\title{
INTÉRÊT ET LIMITES DU TEST STREPTOCOCCUS ZYMOGENES COMME MÉTHODE DE ROUTINE POUR LE CLASSEMENT D'ALIMENTS PROTIDIQUES DE MEME ORIGINE
}

\author{
R. JACQUOT, J. ABRAHAM, J. PERETIANU, R. FERRANDO, Nicole HENRY \\ avec la collaboration de Ime $^{\mathrm{m}}$ de SaINTAURIN \\ Centre de Recherches sur la Nutrition du C.N.R.S. 92 - Bellevue \\ École nationale vétérinaire, \\ Service de Nutrition et d'Alimentation 94 - Alfort \\ Institut national de la Recherche agronomique
}

\section{SOMIMAIRE}

Description de la méthode microbiologique de Ford (Streptococcus zymogenes) avec des modifications originales. Cette technique donne des résultats en bonne concordance avec les données obtenues invivo sur le Rat et le Poulet lorsqu'il s'agit de produits d'origine animale (farines de viande et de poisson non traitées par des agents chimiques tels que le $\mathrm{CCl}_{4}$ ). Par contre elle ne s'applique pas au tourteau de soja.

\section{IN'TRODUC'TION}

Uifférents moyens d'évaluation de la valeur nutritive des protéines ont été expérimentés depuis de nombreuses années. Dès I9I9 OSBORNE, MENDEL et FERRY proposaient une méthode basée sur la croissance du Rat (Coefficient d'Efficacité Protéique: Protein Efficiency Ratio). Depuis, ces essais ont été très souvent repris sous une forme plus ou moins modifiée. L'un de nous a récemment fait le point sur la question (JACQUOT et al., I966). Toutes les techniques utilisant le réactif animal ont l'inconvénient d'être longues (8 jours au minimum pour la plus rapide). De plus, elles sont laborieuses (nombre élevé d'animaux) et exigent un abondant matériel alimentaire.

On a donc cherché à substituer des tests in vitro aux essais sur animaux. La valeur nutritionnelle des protéines dépend au premier chef de leur composition en acides aminés. On dispose actuellement d'un choix de moyens permettant de doser les amino -acides par chromatographie ou microbiologie. Si elles ont fait leurs preuves dans des laboratoires spécialisés, ces méthodes ne sauraient être utilisées dans la 
pratique courante. En outre, elles mettent en œuvre une hydrolyse préalable au dosage et, de ce fait, ne renseignent pas sur la "disponibilité " des acides aminés. I1 existe bien une technique chimique de détermination de la lysine disponible (CARPENTER, I 960), mais son intérêt est limité du fait même de sa spécificité.

Les contrôles de routine font appel à des tests in vitro adaptés à un matériel protidique bien défni: dosage de l'acidité, de l'ammoniac, de l'hydroxyproline et digestibilité pepsique pour les farines animales; activités uréasique et anti-trypsique, fixation de colorants, solubilité dans l'eau ou la soude diluée pour le soja. Ces procédés sont d'un usage facile mais leur simplicité les rend peu sélectifs et leur portée est généralement limitée à l'élimination de produits particulièrement défectueux.

Les méthodes microbiologiques semblent associer les avantages pratiques des tests in vitro à la signification nutritionnelle de l'expérimentation in vivo puisque le plus souvent elles ne nécessitent pas l'hydrolyse des protides alimentaires.

Elles ont débuté avec les travaux de l)unn et Rockiand (I947) à l'aide de Tetrahymena geleii puis se sont développées en faisant appel à d'autres microorganismes.

La méthode de Forv ( 1960 ) semble particulièrement intéressante en raison des besoins que manifeste le microorganisme qu'elle utilise: Streptococcus zymogenes. Celui-ci exige pour sa croissance 8 acides aminés dont 7 sont indispensables pour les animaux supérieurs (arginine, histidine, isoleucine, méthionine, leucine, tryptophane et valine), le $8 \mathbf{e}$ étant l'acide glutamique. Sans être strictement indispensables, la lysine comme la sérine exercent un effet favorable. D'après son auteur, ce test permet de classer les protéines selon un index qui présente une bonne corrélation avec la hiérarchie obtenue sur l'animal. Ultérieurement, FoRv (I962) a élargi sa technique à l'estimation de la disponibilité des sept acides aminés indispensables précités ; en effet, Streptococcus zymogenes possède une forte activité protéolytique qui permet d'éviter l'hydrolyse chimique de l'échantillon à doser.

L'objet de ce travail est d'exposer l'intérêt et les limites de cette méthode en vue d'applications de routine au sein d'un même groupe d'aliments protidiques. Nos résultats se rapportent aux tourteaux de soja, aux farines de poisson et aux farines de viande soit du commerce, soit préparées au laboratoire. Des essais parallèles sur le Poulet et le Rat permettent de confronter le classement fourni par l'index microbiologique et celui que donne la croissance des animaux.

\section{MATÉRIEL E'T MÉTHODES}

Certaines modifications apportées au protocole initial de FoRD ont été rappelées par Boyne et al. ( $\left(9_{67}\right)$ : elles portent surtout sur l'homogénéisation de l'échantillon et sur la prédigsstion enzymatique (augmentation de la quantité de papaine). D: notre côté, nous avons utilisé (ABraifaM et al., $\left.\mathrm{I}^{6} 6_{3}\right)$ une attaque pepsique analogue ì celle qu'on emploie pour l'étude de la digestibilité in vitro des farines animales, suivie d'une digestion trypsique dérivée de celle utilisée pour la mesure de l'activité anti-trypsiçue du tourteau de soja.

\section{Préparation de l'échantillon}

En principe l'échantillon testé doit passer totalement au tamis 40 (Afnor 28). Si besoin est, il faut traiter le refus au broyeur en évitant tout échauffement. Nous avons supprimé cette préparation pour les farines de viande qui contenaient un fort pourcentaga d'os et de tendons en estimant 
qu'il était préférable de tester l'échantillon tel qu'il se présentait plutôt que de risquer d'éliminer une partie importante du matériel de moindre valeur biologique, ce qui aurait finalement faussé la mesure. Par contre, le tamisage a été maintenu pour les farines de poisson et le soja.

\section{Prise d'essai}

La prise d'essai doit contenir too $\mathrm{mg}$ d'azote $(625 \mathrm{mg}$ de protéine). Il est nécessaire d'avoir procédé auparavant au dosage de l'azote total (méthode de Kjeldhal) en éliminant éventuellement du calcul l'azote ammoniacal et l'azote uréique quand il en existe en grande quantité.

\section{Protéine de référence}

On utilise comme protéine de référence la caséine "Difco Vitamine free"; elle sert de témoin pour chaque série de dosages.

\section{Attaque enzymatique}

a) Digestion pepsique.

La prise d'essai est introduite dans une ffole conique de $50 \mathrm{ml}$ avec $20 \mathrm{mg}$ de pepsine Difco (titre $\mathrm{r} / \mathrm{10} 000), 20 \mathrm{ml} \mathrm{d}$ 'HCl $(d=\mathrm{I}, 180)$ à $\mathrm{I}, 25 \mathrm{p}$. I00 (v/v dans l'eau bidistillée). Les foles sont bouchées et placées dans un bain-marie à $37^{\circ} \mathrm{C}$ avec agitation magnétique pendant $3 \mathrm{~h} .3^{\circ}$.

b) Digestion trypsique.

En utilisant un pIImètre ou du papier indicateur de $\mathrm{pH}$, on ajuste à 8,4 le $\mathrm{pH}$ du contenu de la fiole à l'aide de soude et d'acide orthophosphorique; on introduit alors $20 \mathrm{mg}$ de trypsine Merck (40 000 unités Fuld Gross au g); on bouche et on porte à nouveau au bain-marie à agitation à $37^{\circ} \mathrm{C}$ pendant 2 heures.

\section{Milieu de base}

$1^{\circ}$. Préparation de la solution de sels minéraux entrant dans la composition du milieu de base.

$\begin{array}{lc}\quad \text { (Pour I litre de solution) } \\ \mathrm{MgCl}_{2} 6 \mathrm{H}_{2} \mathrm{O} & 2 \mathrm{O} \\ \mathrm{CaCl}_{2} & 5 \\ \mathrm{FeCl}_{3} 6 \mathrm{H}_{2} \mathrm{O} & 0,5 \\ \mathrm{ZnSO}_{4} 7 \mathrm{HO} & 0,5 \\ \mathrm{MnSO}_{4} 4 \mathrm{H}_{2} \mathrm{O} & 0,25 \\ \mathrm{CoCl}_{2} 6 \mathrm{H}_{2} \mathrm{O} & 0,25 \\ \mathrm{VSO}_{4} & 0,25 \\ \mathrm{Na}_{9} \mathrm{MoO}_{4} & 0,25 \\ \mathrm{CuSO}_{4} 5 \mathrm{H}_{2} \mathrm{O} & 0,25\end{array}$

Tous ces sels sont dissous dans I litre d'eau bidistillée en ajoutant 8 à ro $\mathrm{ml}$ d'acide sulfurique normal pour dissoudre un éventuel précipité. Cette solution se conserve plusieurs mois au réfrigérateur à $+4^{\circ} \mathrm{C}$. Le léger dépôt qui peut se produire au fond du flacon est sans inconvénient.

$2^{\circ}$. Composition du milieu de base.

(pour un litre de milieu)

\begin{tabular}{|c|c|c|c|}
\hline Glucose & $60 \mathrm{~g}$ & Pyridoxal & Io $\mathrm{mg}$ \\
\hline Phosphate bipotassique & $30 \mathrm{~g}$ & (pyridoxal éthylacétate hydrochloride) & \\
\hline Acide citrique & $2,5 \mathrm{~g}$ & Riboflavine & Io $\mathrm{mg}$ \\
\hline Acétate de sodium trihydraté & $\mathrm{I} 2,5 \mathrm{~g}$ & Acide nicotinique & Io $\mathrm{mg}$ \\
\hline Tween $80\left(^{(1)}\right.$ & $5 \mathrm{ml}$ & Pantothénate de calcium & to $\mathrm{mg}$ \\
\hline Solution sels minéraux & $50 \mathrm{ml}$ & Acide folique & I $\mathrm{mg}$ \\
\hline Adénine & $25 \mathrm{mg}$ & Acide paraminobenzoïque & Io $\mathrm{mg}$ \\
\hline Guanine & $25 \mathrm{mg}$ & Biotine & $50 \gamma$ \\
\hline Uracile & $25 \mathrm{mg}$ & Vitamine $\mathbf{B}_{12}$ & Io $\dot{\gamma}$ \\
\hline Xanthine & $25 \mathrm{mg}$ & Acide ascorbique & $2,5 \mathrm{~g}$ \\
\hline Thiamine & $10 \mathrm{mg}$ & & \\
\hline
\end{tabular}

(1) Polyoxyśthylène sorbitan mono-oléate.

Annales de Zootechnic. - 1968. 
Le milieu de base stocké en flacon de polyéthylène, se conserve plusieurs mois à $-20^{\circ} \mathrm{C}$ ou I mois $\dot{a}+4^{\circ} \mathrm{C}$.

\section{Méthode de prépation du milieu de base}

a) Dissoudre à froid avec une petite quantité d'eau bidistillée clans un ballon à fond plat de 3 litres le glucose, le phosphate bipotassique, l'acide citrique, l'acétate de sodium et l'acide ascorbique ;

b) dans un bécher de $250 \mathrm{ml}$, chauffer légèrement un peu d'eau bidistillée et y dissoudre les vitamines (sauf $\mathrm{B}_{2}$ et acide folique) à l'abri de la lumière ;

c) dans un bécher, dissoudre l'acide foli fue dans roo $\mathrm{ml}$ d'eau bidistillée chaude ;

d) dans un autre bécher, dissoudre la vitamine $B_{2}$ dans $200 \mathrm{ml}$ d'eau chaude à l'abri de la lumière;

e) diluer le 'Tween 80 dans environ $100 \mathrm{ml}$ d'eau, y ajouter les sels minéraux ;

f) dissoudre les bases puriques en chauffant doucement dans 5 à $6 \mathrm{ml}$ d'acide chlorhydrique à 25 p. too $(\mathrm{v} / \mathrm{v})$, cette dissolution est assez lente ;

g) dissoudre la xanthine dans $200 \mathrm{ml}$ d'eau bidistillée en ajoutant 2 à 5 gouttes de $\mathrm{NaOH}$ ro $\mathrm{N}$ et en chauffant légèrement si besoin ;

h) introduire ces différentes solutions, dans l'ordre, dans la fiole de 3 litres, agiter soigneusement sans secouer ;

i) ajuster le pH à 7,2 ; au pII mètre ou au papier indicateur, à l'aide de soude ou d'acide orthophosphorique.

j) transvaser dans une fiole d'un litre, ajuster au volume à l'aide d'eau bidistillée.

\section{Dosage propremenl dit}

A l'aide d'acide orthophosphorique et, éventuellement, de soude, on ajuste à 7,2 le $\mathrm{pH}$ du contenu de la fiole où a eu lieu la digestion trypsiı [ue de la caséine étalon ou de l'échantillon. On transvase ensuite dans un ballon jaugé de $100 \mathrm{ml}$ et on complète au volume à l'aide d'eau bidistillée. On opère alors une dilution au vingtiène (par exemple $10 \mathrm{ml}$ dans 200) dans une solution aqueuse de glutamate de sodium ì $0,026 \mathrm{p}$. гоo. On prélève des quantités croissantes de cette dernière dilution par exemple $2,4,6,8, \mathrm{ml}$ que l'on introduit, chacune, dans un tube à essai contenant $2 \mathrm{ml}$ de milieu de base. Chaque prise est répétée trois fois. On ajoute de l'eau bidistillée pour obtenir un volume uniforme de $10 \mathrm{ml}$.

Les tubes sont bouchés individuellement avec du coton ou capsulés d'aluminium ou protégés collectivement contre les contaminations ultérieures à l'aide d'un couvercle métallique très embồtant sur chaque portoir. On les porte 5 minutes à l'autoclave à $100^{\circ}$ puis on les refroidit à $37^{\circ}$. On ajoute alors dans chacun d'eux, avec les précautions d'usage, une gout te et une seule d'inoculum de Streptococcus zymogenes. L'incubation se fait à l'étuve à $37^{\circ} \mathrm{C}$ pendint 44 heures.

Fn fin d'incubation, les tubes sont autoclavés ì $100^{\circ} \mathrm{C}$ ' pour arrêter toute évolution du microorganisme pendant la durée du dosage turbidimétrique. Après refroidissement, on agite doucement chaque tube avant de passer au colorimètre à $5600 \AA$.

\section{Lixpression des résultats}

On reporte graphiquement la croissance de Streptococcus zymogenes exprimée par la densité optique en fonction de la concentration du milieu en protéines à juger. On donne la valeur de roo à la surface comprise entre la courbe représentative de la caséine et la ligne de base. Par comparaison avec cette surface. la planimétrie des aut res courbes permet de donner une valeur à chacune des protéines étudiées.

\section{Choiv et détermination de la ligne de base}

Théoriquement, pour chaque concentration de chaque protéine, la croissance de Streptococcus zymogenes doit s'estimer par la différence de densité optique entre le milieu ensemencé et un même tube non ensemencé puisque le résultat de la digestion enzymatique est toujours un liquide légèrement trouble. Il faudrait donc prévoir autant de "blancs " que de tubes ensemencés. Én réalité, quand on se limite au tourteau de soja et aux farines de viande et de poisson, il n'y a praticjuement pas de différence entre les tubes restés stériles; c'est pourquoi un seul point suffit généralement pour déterminer la position de la ligne de base.

\section{Entretien de la souche et préparation de l'inoculum}

La souche de Streptococcus zymogenes a été fournie par la National collection of Dairy Organisms at the National Institute for Research in Dairying, Shinfield-Reading, Berkshire, Grande-Bretagne. Flle est identifiée sous le no ${ }^{0} \mathrm{NCDO} 592$. 
Cette souche lyophilisée est d'abord repiquée deux jours de suite sur milieu liquide (glucose $2 \mathrm{~g}$. extrait de levure Difco $2 \mathrm{~g}$, lait peptonisé Difco $2 \mathrm{~g}$, dissous à chaud dans de l'eau bidistillée q.s. pour $100 \mathrm{ml}$, autoclavage $\mathrm{I} 0$ minutes ì $\mathrm{I} 20^{\circ} \mathrm{C}$. La conservation de ce milieu est de 2 mois à l'abri de la lumière).

Si les dosages ont lieu épisodiquement, la souche est conservée sur milieu gélose (extrait de levure Difco $2 \mathrm{~g}$, glucose anhydre $0,5 \mathrm{~g}$, acétate de sodium $0,5 \mathrm{~g}$, gélose Bacto-Agar Difco I, $5 \mathrm{~g}$, eau bidistillée q.s.p. Ioo ml, autoclavage de ro minutes à $\mathrm{I} 20^{\circ} \mathrm{C}$ ) avec ensemencement par piquure. Ia culture se développe pendant 24 heures à l'étuve à $37^{\circ} \mathrm{C}$ et on la conserve à $+4^{\circ}$.

Deux jours avant le dosage on ensemence, à partir de la culture sur gélose, un tube contenant $5 \mathrm{ml}$ du milieu liquide décrit précédemment ; on fait incuber à $37^{\circ} \mathrm{C}$ pendant 24 heures puis on repique sur le même milieu liquide. Au bont de 24 heures, on ajoute $5 \mathrm{ml}$ de sérum physiologique (ClNa 9 p. I ooo dans de l'eau bi-distillée stérile). L'inoculum est prêt ă l'emploi.

\section{RÉSULTATS}

Les essais poursuivis ont porté sur trois types de produits alimentaires à forte teneur en protéine : tourteaux de soja, farines de poisson et farines de viande.

\section{Tourteaux de soja}

La qualité clu tourteau de soja dépend de la nature et de l'intensité du traitement thermique qu'il a subi. Il faut que la cuisson le débarrasse des facteurs antinutritionnels que contient la graine, mais tout excès de chaleur devient préjudiciable en altérant les protéines. Comme il a été dit, on dispose d'un large choix de tests in vitro qui prétendent renseigner sur son degré de cuisson. Mais les corrélations ne sont pas toujours étroites entre les résultats de ces tests (ABRAHAM, I964; JAcquoT et al., I965; ZEI,TER, I965) et, à plus forte raison, entre techniques in vitro et in vivo. Nous avons voulu voir si la méthode microbiologique pouvait éclairer la question.

Nous avons simultanément étudié avec le test microbiologique et sur animaux, 2 I tourteaux du commerce délipidés et cuits. Les essais sur poulets ont été réalisés à la station expérimentale de 1'U. F. A. C. (Vigny). Il s'agissait d'animaux pesant au départ $200 \mathrm{~g}$ répartis en lots de $\mathrm{I} 6$ sujets par régime et placés en cages individuelles. Les rations équilibrées en minéraux et vitamines étaient du type semi-synthétique; 1'apport protéique, de I 8 p. Ioo, provenait uniquement du tourteau de soja testé. Comme protéine de référence, on a choisi le tourteau $n_{1}^{0} 2$ I qui avait fourni les meilleures performances dans des essais poursuivis antérieurement. On a donné la valeur arbitraire de roo à la croissance réalisée par ce lot dans chaque essai comparatif et exprimé par rapport à cette référence les croissances des autres lots. Les résultats sont rassemblés dans le tableau $\mathrm{I}$.

Il n'existe aucune corrélation entre les deux tests comme le montre bien la dispersion des points de la figure I.

Les indications fournies par la méthode microbiologique telle que nous l'avons utilisée ne semblent pas exploitables pour le classement de la valeur nutritionnelle des tourteaux de soja du commerce. Malgré leur imprécision, les méthodes classiques gardent donc ici leur intérêt.

\section{Farines de poisson}

C'est dans un but uniquement pratique que nous désirions savoir si la méthode microbiologique présentait un avantage par rapport aux procédés simples, presque empiriques, utilisés classiquement pour donner un ordre de grandeur de la qualité 
R. JACQUOT ET COLL.

TABLEAU I

Essais comparés avec les tourteaux de soja

\begin{tabular}{|c|c|c|}
\hline $\begin{array}{c}\text { Tourteaux } \\
n^{0}\end{array}$ & E. P. (1) & I. M. $\left(^{2}\right)$ \\
\hline 1 & 68 & 99 \\
\hline 2 & 74 & 91 \\
\hline 3 & 75 & 91 \\
\hline 4 & 77 & 99 \\
\hline 5 & 85 & 100 \\
\hline 6 & 86 & 95 \\
\hline 7 & 86 & 103 \\
\hline 8 & 88 & 101 \\
\hline 9 & 91 & 89 \\
\hline 10 & 91 & 91 \\
\hline 11 & 92 & 89 \\
\hline 12 & 95 & 87 \\
\hline 13 & 95 & 91 \\
\hline 14 & 95 & 95 \\
\hline 15 & 95 & 96 \\
\hline 16 & 96 & 89 \\
\hline 17 & 97 & 91 \\
\hline 18 & 97 & 101 \\
\hline 19 & 98 & 94 \\
\hline 20 & 100 & 94 \\
\hline Référence 21 & 100 & 95 \\
\hline
\end{tabular}

(1) E. P. : Efficacité protéique (gain de poids du poulet).

(2) I. M. : Indice microbiologique (croissance de Streptococcus zymogenes).

FIG. I. - Tourteaux de soja

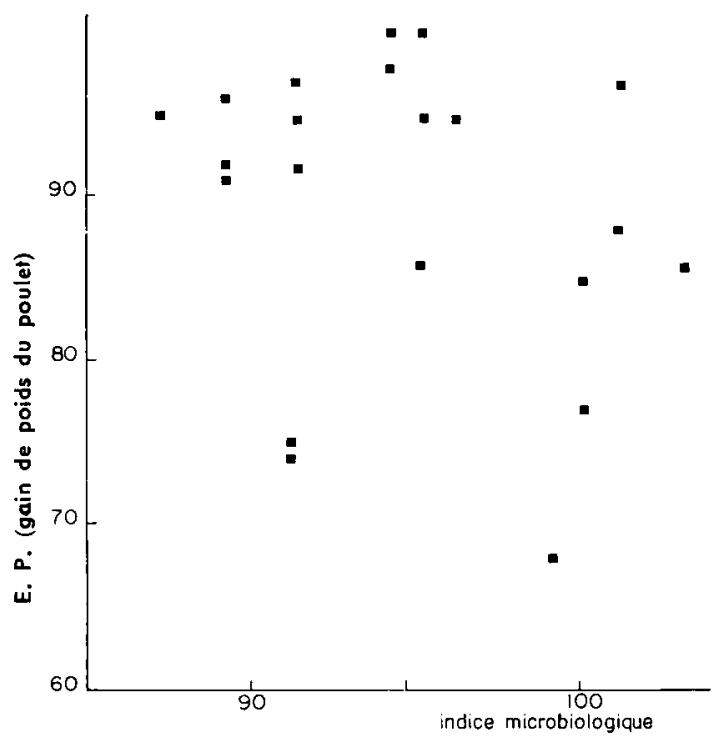


des farines de poisson, à savoir : solubilité pepsique, acidité, teneur en ammoniac, qui complètent habituellement la détermination des protéines totales, des lipides, des matières minérales et de leur fraction insoluble dans l'acide chlorhydrique. Dans un marché en voie de normalisation, ces méthodes ne sauraient être utilisées pour classer les farines de poisson en fonction de leur efficacité protéique, mais simplement pour éliminer des produits particulièrement déclassés. Cela se produit rarement: c'est ainsi que sur $2 \mathrm{I}$ farines étudiées, seul l'échantillon $n^{\circ} \mathrm{I}$ aurait été refusé du fait de sa solubilité pepsique particulièrement faible.

L'examen de ces farines a été conduit dans les mêmes conditions que celles décrites pour le soja en utilisant, cette fois, comme protéine de référence, la farine de hareng $n^{0} \mathrm{I} 8$. Les résultats comparatifs sont rapportés dans le tableau 2.

TABIEAU 2

Essais comparés avec les farines de poisson

\begin{tabular}{|c|c|c|}
\hline $\begin{array}{c}\text { Farines } \\
\mathrm{n}^{0}\end{array}$ & E. P. (1) & I. M. $\left({ }^{2}\right)$ \\
\hline 1 & 51 & 62 \\
\hline 2 & 55 & 76 \\
\hline 3 & 59 & 90 \\
\hline 4 & 60 & 105 \\
\hline 5 & 77 & 80 \\
\hline 6 & 87 & 84 \\
\hline 7 & 90 & 95 \\
\hline 8 & 91 & 82 \\
\hline 9 & 93 & 93 \\
\hline 10 & 93 & 96 \\
\hline 11 & 95 & 66 \\
\hline 12 & 95 & 86 \\
\hline 13 & 95 & 96 \\
\hline 14 & 96 & 101 \\
\hline 15 & 97 & 95 \\
\hline 16 & 97 & 102 \\
\hline 17 & 100 & 99 \\
\hline Référence 18 & 100 & 100 \\
\hline 19 & 100 & 100 \\
\hline 20 & 104 & $12:$ \\
\hline 21 & 106 & 116 \\
\hline
\end{tabular}

(1) E. P. : Efficacité protéique (gain de poids du poulet).

(12) I. M. : Indice microbiologique (croissance de Sireptococcus zymogenes).

La méthode microbiologique fournit des données intéressantes qui varient dans le même sens que celles obtenues sur le Poulet. Néanmoins, le parallélisme n'est pas toujours parfait : ainsi les farines 3 et 4 ont un indice microbiologique élevé alors que leur efficacité pour l'animal est médiocre. A l'inverse, l'échantillon II présente un index microbiologique faible mais une efficacité nutritionnelle satisfaisante. Afin de souligner l'aspect pratique de cette étude, nous avons pensé, en première approximation, pouvoir ne retenir que les I 5 farines ayant des index in vivo et in vitro supérieurs 
à 85. Dans ces conditions, la corrélation entre ces index est excellente $(r=0,85)$ et la régression linéaire suivante les relie :

$\mathrm{Y}=0,4 \mathrm{I} \mathrm{X}+5^{6}\left\{\begin{array}{l}\mathrm{Y} \text { représente le gain de poids relatif du poulet, donc la valeur } \\ \text { nutritionnelle, et } \\ \mathrm{X} \text { 1'index microbiologique. }\end{array}\right.$

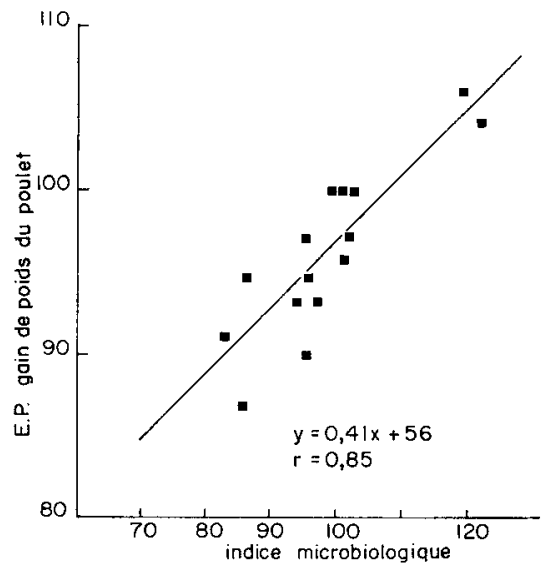

FIG. 2. - Farines de poisson

(Graphique limité aux résultats obtenus sur poulet et pour des indices in vivo et in vitro $>85$ )

Cette simplification peut paraître arbitraire car on aurait pu traiter statistiquement l'ensemble des résultats et calculer ainsi une régression curvilinéaire. Mais pratiquement les farines de faible valeur nutritionnelle sont toujours à éliminer du fait des risques qu'entraîne leur emploi. Seuls ont de l'intérêt les produits de qualité moyenne, bonne ou très bonne, c'est-à-dire ceux qui présentent un index microbiologique supérieur à 85 . Dans ces conditions, nous sommes amenés malheureusement à refuser deux farines convenables ( $n^{\circ} 8$ et $n^{0}$ II) et, en contrepartie, à accepter deux farines de valeur alimentaire médiocre $\left(n^{0} 3\right.$ et $\left.n^{\circ} 4\right)$. Le bilan est néanmoins très positif par rapport aux méthodes traditionnelles qui auraient conduit à l'élimination d'un seul échantillon sans permettre de classer les autres.

Notre comparaison ne s'est pas limitée au poulet. Nous avons également éprouvé le test microbiologique en regard des résultats fournis par la méthode d'alimentation séparée décrite par PERETIANu et ABRAHAM ( 1963 ) pour évaluer la valeur nutritionnelle des protéines chez le Rat. Cinq farines de poisson ont été étudiées et dans quatre cas nous avons trouvé une bonne concordance entre les deux types de techniques.

\begin{tabular}{c|c}
\hline Coefficient d'efficacité protéique ${ }^{1}$ ) & Index \\
\hline (Rat) & \\
85 & 113 \\
87 & 88 \\
98 & 110 \\
104 & 120 \\
105 & 121 \\
\hline
\end{tabular}

() Référence 100 pour la caséine supplémentée en cystine. 
Il arrive que le microorganisme et l'animal réagissent différemment à certains facteurs qui modifient la qualité du produit. C'est ce qui ressort des résultats de Ferrando, Henry et Constantin (i 965) cités au tableau 3.

\section{TABLEAU 3}

Essais comparés avec les farines de poisson

(d'après Constantin, 1964)

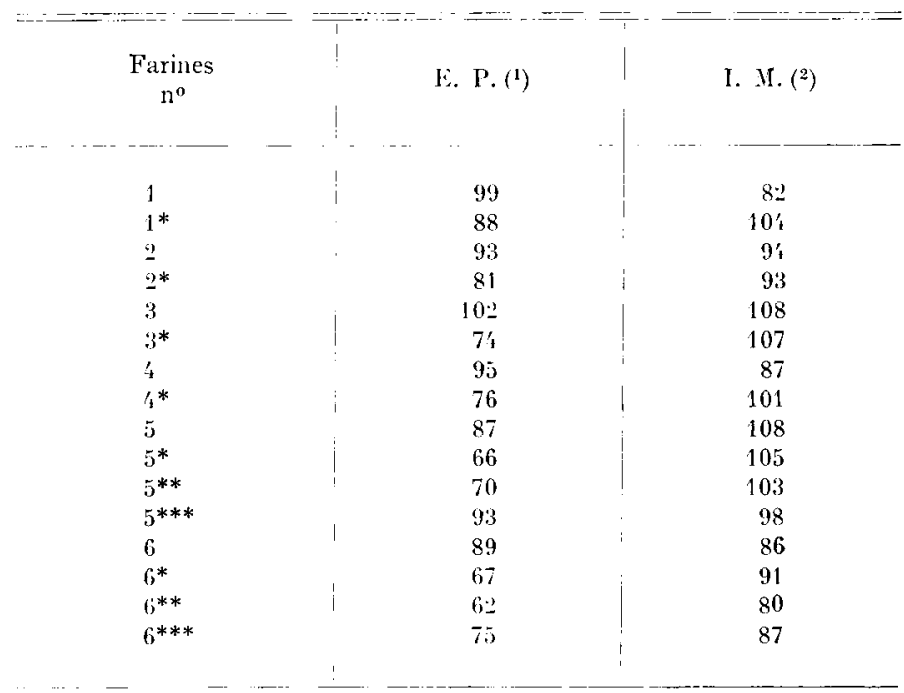

* Farine ayault subi une décantation au $\mathrm{CCl}_{4}$.

** Farine dégraissée an $\mathrm{CCl}_{4}$.

*** Farine chauffée.

(1) E. P. : Coefficient d'efficacité protéique mesuréée sur le rat, par rajport à un régime à base rle caséine pour 1:21\% 100 de proténes

${ }^{(2)}$ I. M. : Indice microbiologique (croissance de Sireptocuccus symogenes).

D'après ces résultats on remarque en particulier que la décantation d'une farine dans le tétrachlorure de carbone (1), améliore généralement1'index microbiologique mais déprime l'efficacité protéique mesurée sur 1'animal. Ce point particulier sera discuté à l'occasion de l'étude des farines de viandes.

\section{Farines de viande}

Le but de ce travail a été essentiellement de voir dans quelle mesure le test microbiologique pouvait rendre compte des conséquences de différents traitements dont l'influence sur l'animal avait été préalablement mise en évidence (FERRANDo et al., I962-I968). Parallèlement aux essais sur Streptococcus zymogenes, 1'efficacité protéique fut mesurée sur le Rat.

(1) Le $\mathrm{CCl}_{4}$ était destiné à éliminer les arêtes de valeur nutritive moindre.

D'après Ferrando et Henry, (ig68). C'est à partir de ce tableau que nous avons calculé le coefficient de corrélation $r=+0,895$. 
Les farines de viande étudiées, ou la caséine lactique servant de protéine de référence, furent introduites de façon à apporter dans le régime I3 p. Ioo de matières protéiques. L'apport glucidique fut réalisé sous forme de saccharose. Les régimes étaient équilibrés en minéraux et vitamines selon le protocole décrit en I 962.

Les matières premières utilisées correspondaient soit à des farines préparées de manière artisanale au laboratoire, soit à des produits du commerce, soit à des farines préparées de façon semi-industrielle, soit enfin à des farines de cretons.

Cinquante farines de viande ou de cretons ont subi 1'essai avec Streptococcus zymogenes. Parmi elles, vingt-neuf ont été distribuées à des rats. Les efficacités protéiques ont été établies au cours d'une période de 28 jours sur des rats Wistar mâles, pris au sevrage, provenant de l'élevage du laboratoire et pesant en début d'expérience 40 à 42 grammes. Pour les résultats du tableau 5 , tous les essais sur animaux n'ont pas été accompagnés du lot témoin caséine, ce qui réduit sensiblement les résultats exploitables. On peut cependant admettre, à la suite des nombreuses mesures d'efficacité protéique, que la réponse moyenne des animaux du laboratoire d'Alfort à un régime contenant $I 3 \mathrm{p}$. Ioo de caséine, est : $r, 93$ 上 0,29 .

Les farines de viande utilisées ont parfois subi des traitements inhabituels (séjour dans l'alcool ou sous $\mathrm{NH}_{3}$ ) tels que l'index microbiologique demeure d'environ 80 , alors que l'efficacité protéique est nulle. Ces traitements ont été tentés afin de déterminer si leur application, qui faciliterait le transport des équarrissages à une usine centrale de traitement en satisfaisant aux exigences sanitaires (dénaturation en vue de les rendre impropres à la consommation humaine), pouvait influencer la valeur nutritive des farines de viande. Nous avons donc été amenés à supprimer certains résultats avant d'essayer d'établir une relation susceptible d'exister entre l'index microbiologique et l'efficacité protéique sur rat (FERRAndo et al., I968).

Les résultats comparatifs figurent aux tableaux 4 et 5 et à la figure 3 tels que nous les avons publiés par ailleurs (1968) pour le tableau 4 et la figure 3 .

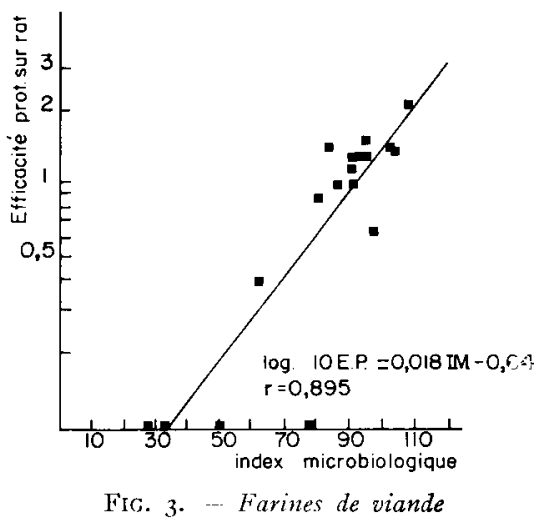

Avec les farines à l'état brut on observe un parallélisme entre le test Streptococcus zymogenes et l'efficacité protéique mesurée sur le rat. Les écarts augmentent considérablement lorsqu'on juge de la même façon les farines après traitement au $\mathrm{CCl}_{4}$. La décantation semble entraîner, à l'exception d'un seul échantillon, une légère valorisation de la qualité des protéines déterminée à l'aide de Streptococcus zymogenes 
TABI,EAUX 4 et 5

Essais comparés avec les farines de viande et de tendons

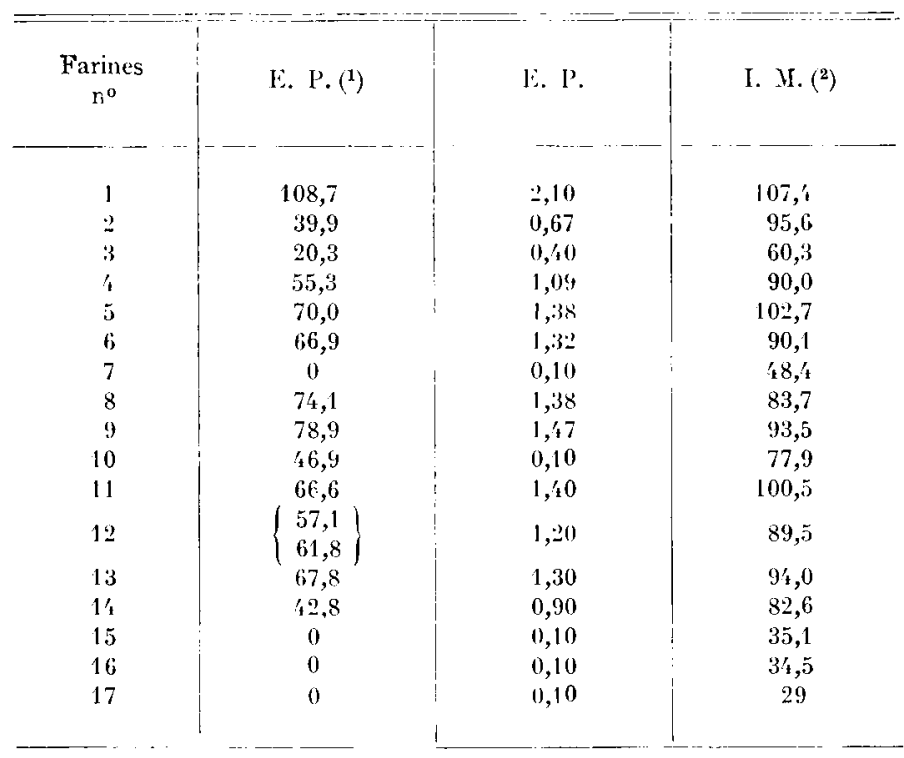

(1) F. P. : Coefficient d'efficacité protéique mesuré sur le rat en pourcentage par rapport à l'efficacité protéique de la caséine.

$\left.{ }^{2}\right)$ I. M. : Indice microbiologique (croissance de Streplococcus zymogenes).

D'après Ferrando et Henry, (1968). C'est à partir de ce tableau que nous avons calculé le coefficient de corrélation $r=+0,895$.

Essais comparés avec les farines de viande (V) et de cretons (C)

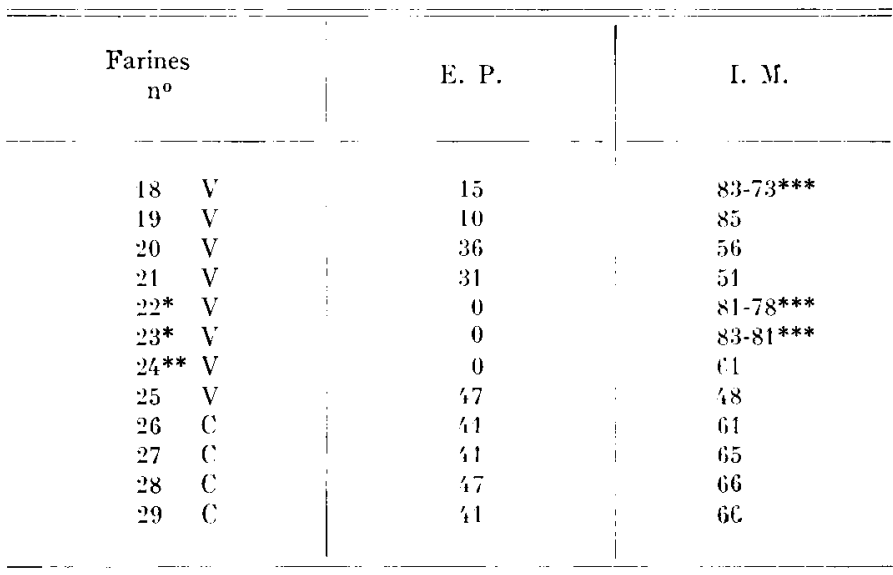

* Viande conservée 8 jours dans l'alcool dénaturé.

** Viande conservée 12 jouts sous atmosphère de $\mathrm{XII}_{3}$.

*** Deux dosages consécutifs.

Le traitement très particulier des échantillons 22,23 et $2 \prime$ enlève, à notre avis, toute valeur à la comparaison. 
alors que le même traitement provoque un effondrement de l'efficacité protéique mesurée sur le rat.

Il apparaît donc que la méthode microbiologique ne donne pas satisfaction dans certaines circonstances, les besoins de Streptococcus zymogenes et sa sensibilité à différents solvants n'étant évidemment pas les mêmes que ceux du rat. Une déficience en méthionine sera, par exemple, fortement ressentie par le rat, elle altérera moins la croissance de Streptococcus zymogenes plus marquée par un manque de thréonine ou de phénylalanine (FORD, I960).

Dans l'ensemble, et quand on considère les échantillons provenant du commerce, on peut cependant admettre que le parallélisme est convenable. Ce qu'on doit souligner, c'est la dissociation totale entre la réponse obtenue chez le mammifère et celle enregistrée avec le microorganisme dans les cas de traitements très spéciaux, tels que décantation dans $\mathrm{CCl}_{4}$ pour quelques farines de poisson ou traitement par $\mathrm{NH}_{3}$ ou l'alcool pour quelques farines de viande. Il s'agit là de traitements expérimentaux mais non habituellement pratiqués dans le cadre de 1'Industrie.

Il est surtout intéressant de constater, qu'au fur et à mesure que diminue la valeur nutritionnelle d'une farine de viande, le développement du microorganisme se ralentit de plus en plus. Certes la réponse n'est jamais égale à zéro comme dans le cas du rat mais, comparativement avec celui-ci, on peut admettre qu'un index microbiologique inférieur à 60 implique une efficacité protéique très médiocre sinon nulle. Ajoutons, à titre documentaire, qu'une série d'essais sur trois farines de viande a permis de montrer l'existence d'un parallélisme entre les réponses de Streptococcus zymogenes, du Rat, du Poulet et du Porc.

\section{CONCLUSION}

Après avoir légèrement modifié (en particulier sur le plan de 1'attaque enzymatique) la méthode microbiologique de Ford basée sur l'utilisation de Streptococcus zymogenes, nous l'avons appliquée à deux types d'aliments riches en protéines, les tourteaux de soja et les farines animales. Dans le premier cas (tourteau de soja), nous n'avons pas pu mettre en évidence de corrélation entre l'index microbiologique et les résultats obtenus sur animaux. Par contre, le test Streptococcus zymogenes semble convenir aux farines de poisson et de viande. Il permet une discrimination plus fine de leur valeur protéique, que les mesures in vitro traditionnellement utilisées, lorsqu'il s'agit de produits de qualité courante. On aboutit ainsi à un classement semblable à celui que donne l'expérimentation sur le Rat ou sur le Poulet.

Cependant, si les échantillons ont subi un traitement particulier ou des altérations notables, on peut observer une discordance importante entre les résultats fournis par le microorganisme et par l'animal.

Une farine de viande ou de poisson n'étant jamais utilisée comme seule source de protéine pour l'alimentation animale, la méthode microbiologique ne saurait renseigner sur les possibilités d'inter-supplémentation entre les diverses protéines. Néanmoins, il est certain que la condition première pour avoir un régime satisfaisant 
est d'utiliser des matières premières de bonne qualité nutritionnelle. A ce point de vue, le test utilisant Streptococcus zymogenes paraf̂t intéressant pour éprouver, de façon simple et rapide, l'efficacité protéique des produits d'origine animale.

Reçu pour publication en juin 1968.

\title{
SUMMARY
}

\author{
THE " STREYTOCOCCUS ZYMOGENES " TEST FOR ASSESSING THE NUTRITIONAL VALUE \\ OF PROTEINS FROM THE SAMF ORIGIN : ITS ADVANTAGES AND LIMITATIONS IN COMMON USE
} test).

The current paper describes a modified Ford's microbiological method (Streptococcus zymogenes

Our results are in definite accordance with those obtained in vivo in the Rat and Chicken for proteins from animal origin (meat and fish meals without chemical treatment with agents such as $\mathrm{CCl}_{4}$ ).

The technique does not work with soybean oil meals.

\section{RÉFÉRENCES BIBLIOGRAPHIQUES}

Abraham J., 1964. Méthodes d'analyses biologiques des tourteaux. Journées d'informalion 1964 sur les produits dèrivés de l'huilerie. No spécial, Rev. F'r. des corps gras, 134-.46.

Abrainay J., Peretiand J., Vie R., 1963. Intérêt et limite d'emploi de la méthode microbiologique de liord pour l'estimation in vitro de la valeur nutritionnelle des proténes. Ann. Biol. anim. Biochim. Biophys. No hors-série, $1,47-48$.

Boyne A. W., PrICe S. A., Rosen G, D., StotT J. A., 1967. Protein quality of feeding stuff. Brit. J. Nuirit., 21, $18 \mathrm{I}-206$.

Carpenter K. J., 1960. 'The estimation of the available lysine in animal Protein Foods. Biochem. J., 7\%, 604-6Io.

Constantin J.-I.., ig64. Étude de l'efficacilé alimentaire des farines de poisson. Thèse I)oct. vétérinaire, Paris.

Dunn M. S., Rockland L. B., I947. Biological value of proteins determined with Tetrahymena geleii $\mathrm{H}$. Proc. Soc. Exp. Biol. Med., 64, 377-379.

Ferrando R., I $96+$. Réaction de Maillard et préparation des farines de viande. Bull. Acad. nat. Méd., $143,570-576$.

Ferrando R., Henry N., Vaiman N., i 962 . Étude sur la valeur alimentaire des farines de viande ( ${ }^{\text {re }}$ et $2^{\mathrm{e}}$ note). Rec. Méd. vét., 133, 379-385 et $457-46 \mathrm{r}$.

Ferrando R., Henry N., Constantin J. J., 1965 . Comparaison de l'efficacité protéique des farines de poisson en utilisant soit le rat, soit Streptococcus zymogenes. Rec. Méd. vét., 141, I37-14I.

Ferrando R., Henry N., Larvor P.. i g68. Relationships between protein efficiency of meat measures rat and thein by means of Streptococcus aymogenes test. Brit. J. Nutrit., 22, I29-1 3I

Ford J. E., 1950. A microbiological method for assessing the nutritionnal value of proteins. Brit. J. Nutrit., 14, 485-497.

FORD J. E., I962. Microbiological method for assessing the nutritional value of proteins. 2. The measurement of "available" methionine, leucine, isoleucine, arginine, histidine, tryptophane and valine. Brit. J. Nutrit., 16, 409-425.

JacQuor R., ABRAHAM J., I965. Influence des traitements industriels et ménagers sur la valeur nutritive des protéines. Nutr. Diet., \%, 43-68.

JACquor R., Rérat A., t966. Ia valeur biologique des protéines. Congrès Mondial d'alimentation animale, p. 329-407, Madrid 1966.

Peretianu J., Abraham J., ig63. Nouvelle méthode de mesure du coefficient d'efficacité protéique. Ann. Nutrit. alim., 17, 81-I02.

Zeeter S. Z., 1965. Estimation des effets de la cuisson des tourteaux d'oléagineux sur la qualité des protéines par des tests biochimiques. Journées d'information 1964 sur les produils dérivés de l'huilerie. $\mathrm{N}$ o spécial, Rev. frans. Corps Gras, 134-1 46. 